\title{
Neonatal respiratory distress syndrome revealing a cervical bronchogenic cyst: a case report
}

\author{
Penelope Thaller ${ }^{1}$, Catherine Blanchett ${ }^{2}$, Maliha Badr ${ }^{1}$, Renaud Mesnage ${ }^{1}$, Nicolas Leboucq ${ }^{3}$, Michel Mondain $^{2}$ \\ and Gilles Cambonie ${ }^{1^{*}}$
}

\begin{abstract}
Background: Bronchogenic cyst is a congenital malformation, rarely located in the cervical region and almost never involved in a neonate with acute respiratory distress in the delivery room.

Case presentation: A female newborn with respiratory distress syndrome caused by a large left cervical mass. Intubation was difficult due to tracheal deviation. Magnetic resonance imaging confirmed a left cervical cyst displacing the trachea and esophagus laterally. Surgical excision was performed via a cervical approach on the 5th day, and pathological examination revealed a bronchogenic cyst. The patient's course was complicated by left vocal cord paralysis and necrotic lesions in the glottic and subglottic regions; she required a tracheostomy on the 13th day. Inflammatory stenosis in the subglottic region required balloon dilation once, 20 days later. Proximal esophageal stenosis induced transient upper airway obstruction with salivary stasis. Decannulation was performed at 2 months and the patient was discharged 10 days later.

Conclusion: A bronchogenic cyst can exceptionally obstruct the airways in the neonatal period. Surgical excision is necessary, but postoperative complications may occur if the cyst is in close contact with the trachea and esophagus, including necrotic and stenotic lesions of the upper aerodigestive tract. In those situations, tracheostomy may be necessary for mechanical ventilation weaning and the initiation of oral feeding.
\end{abstract}

Keywords: Cervical bronchogenic cyst, Neonatal respiratory distress syndrome, Tracheostomy

\section{Background}

Bronchogenic cyst is a congenital malformation of the tracheobronchial tree. It is usually located in the mediastinum or lung parenchyma, though rarely can be seen in extrathoracic locations such as the neck. In 2004, Mehta et al. reported a series of 4 cases of cervical bronchogenic cysts over a 22-year period, with the age of discovery ranging from 3 weeks to 6 years [1]. In 2008, Teissier et al. reported 8 cases over a period of 13 years [2]. The signs and symptoms are variable, but usually consistent with a neck mass associated with chronic cough or stridor [3]. Some cases are not diagnosed until adulthood, when an isolated laterocervical mass is discovered incidentally [4]. We report an exceptional case

\footnotetext{
* Correspondence: g-cambonie@chu-montpellier.fr

'Department of Neonatology and Pediatric Intensive Care Unit, Hôpital Arnaud de Villeneuve, 371 Avenue du Doyen Gaston Giraud, 34295 Montpellier Cedex 5, France

Full list of author information is available at the end of the article
}

of cervical bronchogenic cyst revealed by acute respiratory distress in the neonatal period.

\section{Case presentation}

The patient's mother received prenatal care during pregnancy, with 3 antenatal US exams showing normal fetal development. Labor was induced at $41^{+4}$ gestational weeks in a type 2a maternity ward and delivery was by cesarean section because of abnormal fetal heart rate. The newborn was female with a birth weight of $3045 \mathrm{~g}$. The newborn showed ineffective ventilation from the first postnatal minute, and positive pressure ventilation with a bag and mask was immediately started. A left cervical swelling was also observed. Respiratory distress, with suprasternal tugging and stridor, occurred at every attempt to withdraw ventilatory support. Furthermore, $\mathrm{FiO}_{2}$ of $90 \%$ was required to maintain $\mathrm{SpO}_{2}$ above $90 \%$. Two attempts of orotracheal intubation failed because the larynx could not be visualized. A more experienced pediatrician was called for help. Standard direct 
laryngoscopy found a right laryngeal deviation with a restricted laryngeal orifice. After sedation with midazolam $(0.1 \mathrm{mg} / \mathrm{kg}$ intravenous, 2 doses $10 \mathrm{~min}$ apart), tracheal intubation was achieved at the 3rd attempt, with a 2.5$\mathrm{mm}$ endotracheal tube, at $1 \mathrm{~h} 30 \mathrm{~min}$ after birth. The newborn was then transferred to a type 3 neonatal unit.

On admission, the newborn was ventilated in assistcontrol pressure mode with $\mathrm{FiO}_{2}$ set at $21 \%$. Clinical examination confirmed a noninflammatory cervical swelling that was soft on palpation. Chest $\mathrm{x}$-ray revealed the endotracheal tube deflected to the right with respect to the spinal axis. Cervical US showed a thin-walled cystic mass measuring $24 \times 28 \times 37 \mathrm{~mm}$ displacing the trachea and the left thyroid lobe medially and forward, and the carotid and jugular vessels laterally and posteriorly. MRI identified a large cystic mass with well-defined walls that laterally displaced the aerodigestive tract (Fig. 1).

In the first $48 \mathrm{~h}$ of management, peak inspiratory pressures $>25 \mathrm{~cm} \mathrm{H}_{2} \mathrm{O}$ were necessary to observe chest expansion and hear vesicular breath sounds at auscultation. Volume mode was tested, but it also generated high inspiratory pressures, between 25 and $30 \mathrm{~cm} \mathrm{H}_{2} \mathrm{O}$, despite the selection of a minimal tidal volume $(5 \mathrm{ml} / \mathrm{kg})$ and the use of permissive hypercapnia $\left(\mathrm{PCO}_{2}\right.$ allowed to rise as high as $60 \mathrm{mmHg}$ ). On the assumption that the cyst might be squeezing the endotracheal tube, we performed fine needle aspiration of the cyst in the operating room, under general anesthesia, on postnatal day 3 . Twenty milliliters of clear liquid was removed for cytological analysis, which revealed both respiratory-type epithelium and squamous epithelium. Rigid bronchoscopy performed at the same time confirmed tracheal compression and no laryngeal fistula. A 3-mm endotracheal tube was put into place to secure the airway. On day 5 , surgery using a cervical approach isolated a unilocular cystic structure measuring $25 \times 23 \times 6 \mathrm{~mm}$ in close contact with the trachea and esophagus. Dissection was not possible from the trachea or the esophagus, and limited resection of trachea and esophagus was therefore mandatory, resulting in an opening of both structures. Both were then sutured. Histological examination showed that the cyst wall was composed of fibrous and smooth fibromuscular tissue, with some seromucinous bronchial gland cells. The wall was lined by a layer of respiratory-type ciliated cells. These findings suggested the diagnosis of bronchogenic cyst.

After the first attempt at extubation on the day after surgery, the infant had respiratory distress due to laryngotracheal edema and left vocal fold paresis that was refractory to treatment with nebulized epinephrine and corticosteroids and required reintubation. The second attempt at 8 days postsurgery was equally unsuccessful. The upper respiratory tract was explored under general anesthesia at a postnatal age of 13 days. We observed edema of the left laryngeal ventricle and the anterior third of the glottis, associated with the necrotic appearance of the mucosa in the left lateral subglottis (Fig. 2). Such lesions in a newborn who could not be weaned from mechanical ventilatory support prompted immediate tracheostomy.

Sedation was gradually reduced over $48 \mathrm{~h}$ postsurgery, and mechanical ventilation was ended 2 weeks later with the infant ventilating effectively with the tracheal cannula.

Laryngotracheobronchoscopy under general anesthesia on day 33 revealed a subglottic stenosis treated with a 6-mm diameter balloon dilation. Proximal esophageal stenosis was also observed and not treated. Videographic exploration of swallowing confirmed the images of a very proximal partial esophageal stenosis, from $\mathrm{C} 3$ to $\mathrm{C} 4$.

After a final endoscopic assessment at 2 months showing the absence of airway stenosis, the cannula was withdrawn. The infant was then discharged 10 days later. She was exclusively breastfed and weight and height
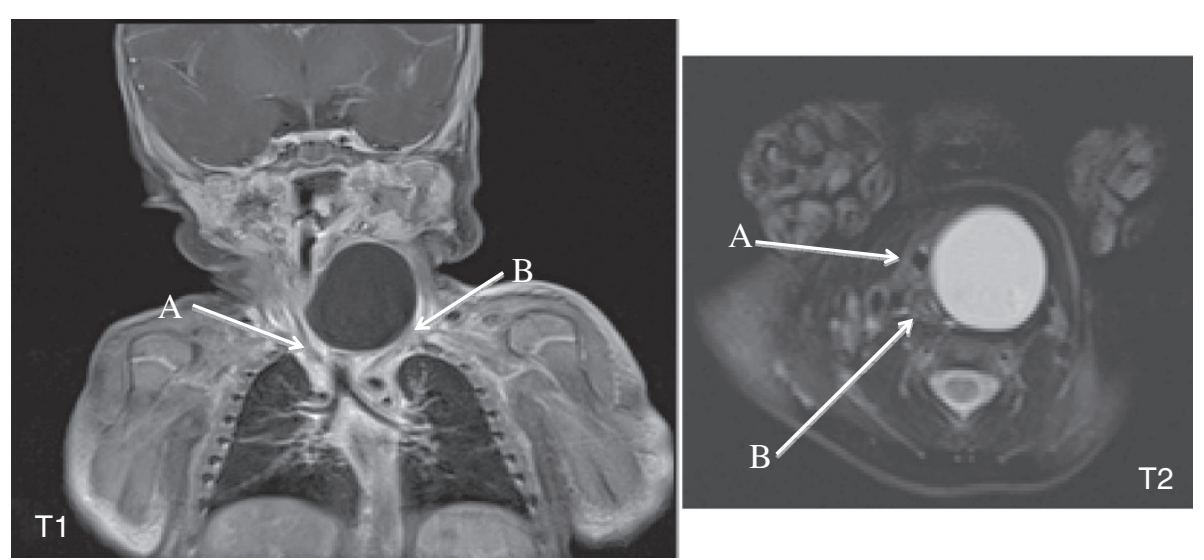

Fig. 1 Cervical thoracic MRI. Coronal T1-weighted sequence showing a voluminous left laterocervical mass displacing the trachea (a) and carotid-jugular axis (b). Axial T2-weighted sequence showing tracheal compression (a) and esophageal compression (b) by the cyst 


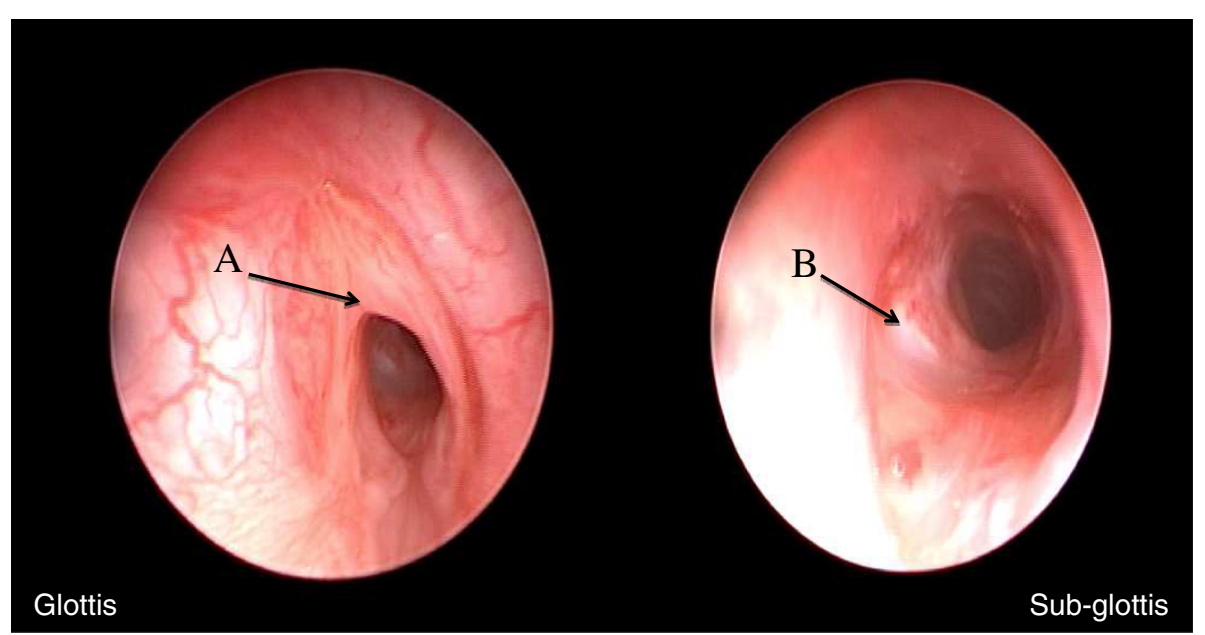

Fig. 2 Postoperative endoscopy. Glottic region: anterior synechia (a) causing luminal stenosis of $50 \%$. Subglottic region: sutured tracheal wound (b) and inflammatory alterations causing grade 1 stenosis

gains were satisfactory, with respective gains of $915 \mathrm{~g}$ and $7 \mathrm{~cm}$ since birth, with absence of swallowing difficulty in relation to the partial esophageal stenosis.

\section{Discussion}

The respiratory bud develops from the ventral side of the primitive foregut from the 4th week of development. Cervical bronchogenic cyst may result from an abnormal division of the bud and migration to an ectopic localization in the neck region $[1,5,6]$. Other extrathoracic localizations have been described, including subcutaneous [7] and even abdominal [8] sites.

Antenatal discovery of a bronchogenic cyst is rare and localization is generally intrathoracic $[9,10]$. One case has been reported of a cervico-mediastinal bronchogenic cyst detected by a cervical mass on prenatal US [11]. Undetected cervical bronchogenic cyst may also cause polyhydramnios by esophageal compression [12]. In both this case and ours, an abnormal fetal heart rate precipitated delivery by cesarean section. Nevertheless, the direct and indirect impact of this malformation on disturbances in fetal monitoring is difficult to establish.

The immediate neonatal respiratory distress of our patient was explained by direct compression of the trachea, causing major difficulties for intubation because the larynx could not be visualized. The use of a videolaryngoscope for intubation can improve the viewing conditions of the upper airways, making it easier to identify the laryngeal inlet. Rigid bronchoscopy or a flexometallic tube can also relieve glottic-subglottic compression, although great caution is required while advancing the scope or the tube. The ex utero intrapartum treatment (EXIT) procedure can also be done if prenatal diagnosis suggests fetal airway obstruction by a neck mass. Its use in this context has mainly been reported for cervical lymphatic malformations and cervical teratomas $[13,14]$.

The main differential diagnosis is cystic hygroma, which is more likely in the setting of a multilocular cyst, and more unlikely in the setting of a unilocular cyst. Other differential diagnoses include thymic cyst, branchial cyst and thyroglossal duct cyst, but they are rarely responsible for neonatal respiratory distress. Only histological examination of the resected specimen confirms the diagnosis.

MRI is essential before surgery, as it provides the surgeon with precise information on the anatomical relationships with adjacent organs [15]. Rigid bronchoscopy assesses the degree of cyst-trachea contact and the need for tracheal surgery [12]. Needle aspiration of the cyst usually has low diagnostic value [16]. It was performed in our patient to temporarily reduce the tracheal compression. However, neither cyst aspiration nor the larger caliber endotracheal tube introduced following bronchoscopy significantly reduced peak inspiratory pressures, which remained high until surgery.

The only treatment is surgical excision, even for an asymptomatic cyst, given the risks of infection, hemorrhage and, rarely, malignant transformation [17]. The paralysis of the left vocal cord may have resulted from direct injury to the laryngeal nerve during surgery. Paralysis was reported in two other cases $[2,12]$, but in one of these cases this condition had already been observed during the preoperative bronchoscopy, suggesting direct mechanical interference between the cyst and the ipsilateral laryngeal nerve [2]. Evaluation of vocal cord mobility before surgery was not possible in our case because of the depth of sedation during bronchoscopy.

Several factors probably contributed to the alterations in the glottic and subglottic regions observed after the 
intervention, including intubation and the repeated attempts to intubate, the inflammation associated with laryngeal surgery, and the prolonged mechanical ventilation. In a newborn, edemic and necrotic lesions further narrow an already physiologically narrow larynx, which is an additional motivation to perform tracheostomy.

In addition to the vocal cord paralysis, persistent airway obstruction after laryngeal surgery should always raise the suspicion of a local cause, such as stenosis. In our patient, a proximal esophageal stenosis was also present after surgery. The damage to the esophageal mucosa was probably caused by the surgery, secondary to cyst resection and the suturing of borders. This stenosis, however, had only transient and moderate clinical consequences and did not preclude satisfactory breastfeeding, even with the tracheal cannula.

\section{Conclusion}

Cervical bronchogenic cyst is rare and diagnosis is most often made in childhood. However, it should be systematically considered in all cases of neonatal cervical tumor, especially if respiratory distress is present. Prenatal diagnosis may be guided by an unexplained polyhydramnios and/or direct visualization of a cyst, in which case delivery should be performed in a maternity hospital with a type 3 neonatal unit. The EXIT procedure, with a multidisciplinary team approach, should also be planned for fetuses with evidence of airway obstruction on prenatal images.

\section{Consent}

Written informed consent was obtained from the parents of the patient for publication of this case report and the accompanying images. A copy of the written consent is available for review by the Editor of this journal.

\section{Abbreviations}

US: Ultrasound; $\mathrm{FiO}_{2}$ : Fraction of inspired oxygen; $\mathrm{SpO}_{2}$ : Oxygen saturation by pulse oximetry; MRI: Magnetic resonance imaging; $\mathrm{Cm} \mathrm{H}_{2} \mathrm{O}$ : centimeter of water $(0.7355 \mathrm{mmHg}) ; \mathrm{PCO}_{2}$ : Partial pressure of carbon dioxide measured on capillary blood gas sampling (mmHg); C3: 3rd cervical vertebra; C4: 4th cervical vertebra.

\section{Competing interests}

The authors declare that they have no competing interests.

\section{Authors' contributions}

PT reviewed the literature and prepared the manuscript, CB helped to draft the manuscript, $\mathrm{MB}$ and $\mathrm{RM}$ made a critical reading of the manuscript and participated to revisions, NL helped to draft the manuscript, MM helped to draft the manuscript and participated to revisions, GC reviewed the literature, prepared the manuscript and is the corresponding author. All authors read and approved the final version of the manuscript.

\section{Acknowledgement}

The authors thank Mrs C Stott-Carmeni for reviewing the manuscript and for editorial assistance.

This case study is from the Neonatal Intensive Care Unit, Arnaud de

Villeneuve Hospital, Montpellier University Hospital Center.

\section{Author details}

'Department of Neonatology and Pediatric Intensive Care Unit, Hôpital Arnaud de Villeneuve, 371 Avenue du Doyen Gaston Giraud, 34295

Montpellier Cedex 5, France. ${ }^{2}$ Department of Pediatric Otorhinolaryngology, Hôpital Arnaud de Villeneuve, 371 Avenue du Doyen Gaston Giraud, 34295 Montpellier Cedex 5, France. ${ }^{3}$ Department of Neuroradiology, CHU

Montpellier, F-34000 Montpellier, France.

Received: 17 October 2014 Accepted: 13 April 2015

Published online: 27 June 2015

\section{References}

1. Mehta RP, Faquin WC, Cunningham MJ. Cervical bronchogenic cysts: a consideration in the differential diagnosis of pediatric cervical cystic masses. Int J Pediatr Otorhinolaryngol. 2004;68:563-8.

2. Teissier N, Elmaleh-Bergès M, Ferkdadji L, François M, Van den Abbeele T. Cervical bronchogenic cysts: usual and unusual clinical presentations. Arch Otolaryngol Head Neck Surg. 2008;134:1165-9.

3. Goswamy J, de Kruiif S, Humphrey G, Rothera MP, Bruce IA. Bronchogenic cysts as a cause of infantile stridor: case report and literature review. J Laryngol Otol. 2011;125:1094-7.

4. Bocciolini C, Dall'olio D, Cunsolo E, Latini G, Gradoni P, Laudadio P. Cervical bronchogenic cyst: asymptomatic neck mass in an adult male. Acta Otolaryngol. 2006;126:553-6.

5. Marks C, Marks P. The embryologic basis of tracheobroncho-pulmonary maldevelopment. Int Surg. 1987;72:109-14.

6. Joshi R, Cobb AR, Wilson P, Bailey BM. Lingual cyst lined by respiratory and gastric epithelium in a neonate. Br J Oral Maxillofac Surg. 2013:51:173-5.

7. Gaikwad P, Muthusami JC, Raj JP, Rajinikanth J, John GM. Subcutaneous bronchogenic cyst. Otolaryngol Head Neck Surg. 2006;135:951-2.

8. El Youssef R, Fleseriu M, Sheppard BC. Adrenal and pancreatic presentation of subdiaphragmatic retroperitoneal bronchogenic cysts. Arch Surg. 2010;145:302-4.

9. Levine D, Jennings R, Barnewolt C, Mehta T, Wilson J, Wong G. Progressive fetal bronchial obstruction caused by a bronchogenic cyst diagnosed using prenatal MR imaging. AJR Am J Roentgenol. 2001;176:49-52.

10. Bernasconi A, Yoo SJ, Golding F, Langer JC, Jaeggi ET. Etiology and outcome of prenatally detected paracardial cystic lesions: a case series and review of the literature. Ultrasound Obstet Gynecol. 2007;29:388-94.

11. Kaji T, Takamatsu H, Noguchi H, Tahara H, Fukushige T, Mukai M, et al. Cervico-mediastinal bronchogenic cyst occurring in the prenatal period: report of a case. Surg Today. 2000;30:1016-8.

12. Cavel O, Kokta V, Reveret M, L'Allier M, Froehlich P, Lapointe A. Subglottic bronchogenic cyst presenting as neonatal asphyxia. Case report and literature review. Int J Pediatr Otorhinolaryngol Extra. 2013;8:92-6.

13. Liechty KW. Ex-utero intrapartum therapy. Semin Fetal Neonatal Med. 2010;15:34-9.

14. Laje P, Peranteau WH, Hedrick HL, Flake AW, Johnson MP, Moldenhauer JS, et al. Ex utero intrapartum treatment (EXIT) in the management of cervical lymphatic malformation. J Pediatr Surg. 2015;50:311-4.

15. Kieran SM, Robson CD, Nosé V, Rahbar R. Foregut duplication cysts in the head and neck: presentation, diagnosis, and management. Arch Otolaryngol Head Neck Surg. 2010;136:778-82.

16. Sumiyoshi K, Shimizu S, Enjoji M, Iwashita A, Kawakami K. Bronchogenic cyst in the abdomen. Virchows Arch A Pathol Anat Histopathol. 1985;408:93-8.14.

17. Ashizawa K, Okimoto T, Shirafuji T, Kusano H, Ayabe H, Hayashi K. Anterior mediastinal bronchogenic cyst: demonstration of complicating malignancy by CT and MRI. Br J Radiol. 2001;74:959-61. 